\title{
Accurate and equitable medical genomic analysis requires an understanding of demography and its influence on sample size and ratio
}

\author{
Michael D. Kessler ${ }^{1,2,3^{*}}$ and Timothy D. O'Connor ${ }^{1,2,3,4^{*}}$
}

The authors of the original article were invited to submit a response, but declined to do so. Please see related Open Letter: http://genomebiology. biomedcentral.com/articles/10.1186/s13059-016-1016-y

\begin{abstract}
In a recent study, Petrovski and Goldstein reported that (non-Finnish) Europeans have significantly fewer nonsynonymous singletons in Online Mendelian Inheritance in Man (OMIM) disease genes compared with Africans, Latinos, South Asians, East Asians, and other unassigned non-Europeans. We use simulations of Exome Aggregation Consortium (ExAC) data to show that sample size and ratio interact to influence the number of these singletons identified in a cohort. These interactions are different across ancestries and can lead to the same number of identified singletons in both Europeans and non-Europeans without an equal number of samples. We conclude that there is a need to account for the ancestry-specific influence of demography on genomic architecture and rare variant analysis in order to address inequalities in medical genomic analysis.
\end{abstract}

Petrovski and Goldstein [1] recently reported on the analysis of a 5965-sample exome sequencing cohort that showed significantly different numbers of nonsynonymous singletons in Online Mendelian Inheritance in Man (OMIM) [2] genes across ancestry groups. More specifically, they showed significantly fewer singletons in Europeans, and they explained this as resulting from what they call a reduced access to ethnically matched

\footnotetext{
* Correspondence: Michael.Kessler@som.umaryland.edu;

TOConnor@som.umaryland.edu

'Institute for Genome Sciences, University of Maryland School of Medicine,

Baltimore, MD 21201, USA

Full list of author information is available at the end of the article
}

controls. When they added 60,252 samples from the Exome Aggregation Consortium (ExAC) reference data set [3] to their analysis, the ancestry-based singleton distributions became more similar but were still significantly different across ancestries. The authors note that although this numerical difference across ancestries in singletons per individual may sound small, it can have a large impact on clinical interpretation and action.

While we concur with their overall conclusions, we would like to highlight that the ancestry-based differences that they observed are more complex and would not be addressed by equal representation across ancestries. Rather, it is important to consider recent demographic differences as they affect the distribution of rare alleles within a population. To demonstrate this, we ran simulations using ExAC allele frequencies of nonsynonymous OMIM [2] disease-gene variants to show that these demographic differences are a function of ancestry sample size and ratio (see Supplementary note in Additional file 1; scripts available upon request). Furthermore, our simulation results are consistent with recent findings about demographic history and allele frequency distribution [4-6]. We compared African, East Asian, South Asian, and Latino samples with (nonFinnish) European samples, and then down-sampled from the ExAC reference cohort to show what candidate variant analysis would look like in studies with diverse cohorts of varying sample sizes. Since our results are qualitatively the same for each of the ancestries when compared with Europeans, we describe the results from our analysis of African and European samples as a representation of the population-based pattern, and present other comparisons in Additional file 1: Figures S1-S6. 
When African and European sample totals are equal, the difference in singletons per individual persists at low sample sizes and is reduced to zero as the number of African and European samples in the analysis cohort each reaches 1000 (Figs. 1 and 2). As these African and European sample totals each reach 5200 (close to the maximum number of African samples in the ExAC reference data set), the number of singletons in Africans becomes significantly lower than the number in Europeans (Figs. 1 and 2). This is consistent with observations from recent large sequencing studies that show that ultralow frequency variants are more prevalent in individuals of predominantly European ancestry than in individuals of predominantly African ancestry as a result of differences in population growth in the past 10,000 years [4-7].

Another key variable is the ratio between African and European sample sizes. As the ratio of African to European sample number decreases, the number of singletons per individual decreases by more in Europeans than in Africans (Fig. 1b). Therefore, when this ratio is low, as is usually the case because of the Eurocentricity of most major sequencing studies, Europeans have a comparatively reduced number of singletons. Our simulation results suggest that researchers usually observe this reduced number of singletons in Europeans compared with Africans as a result of both low sample size ratio and moderate overall sample size (this holds for other less-represented populations as well). Clinically, this becomes a challenging discrepancy that leads to the costly need to adjudicate additional candidate variants in individuals of non-European ancestry $[1,8]$. However, our simulations demonstrate that despite a low ratio of African to European sample size, the difference between populations in singletons per individual goes away as the African population size becomes large enough (Figs. 1 and 2).

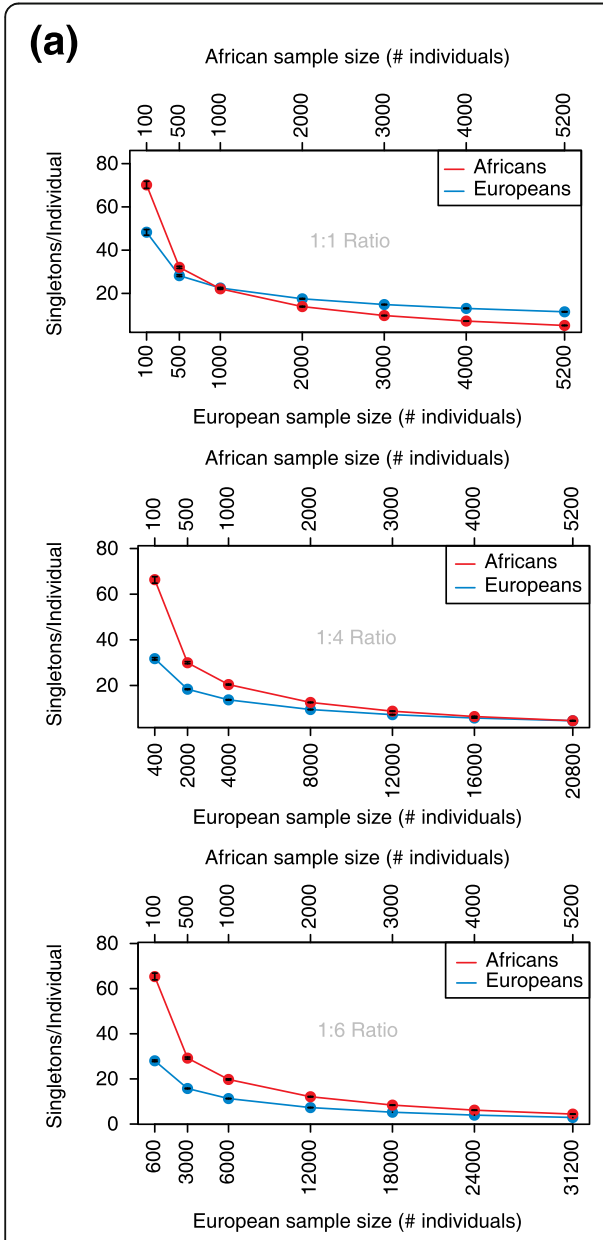

(b)

African sample size (\# individuals)

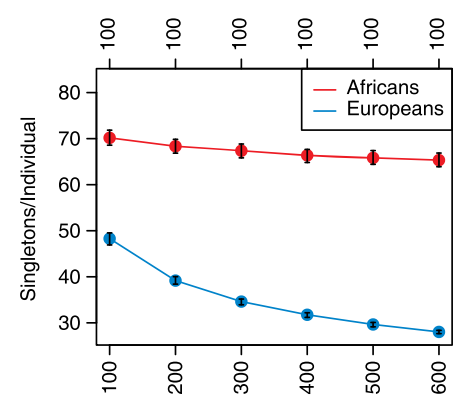

European sample size (\# individuals)

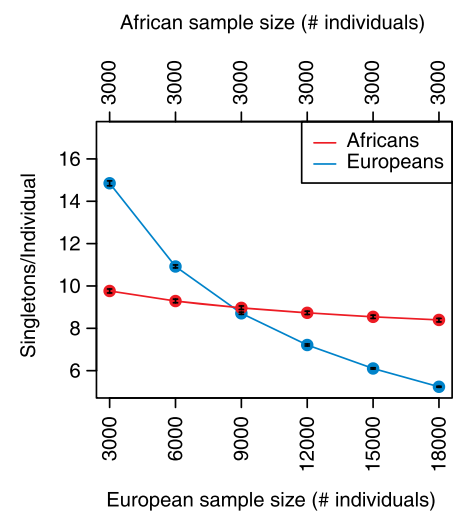

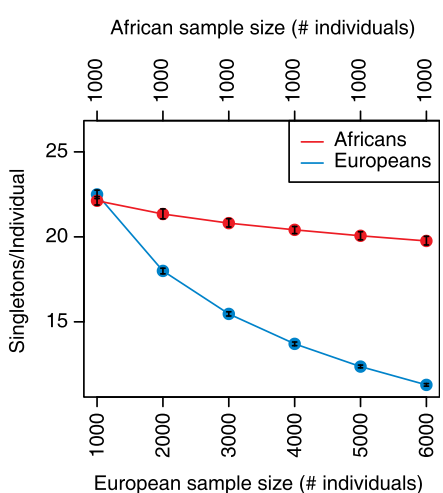

African sample size (\# individuals)

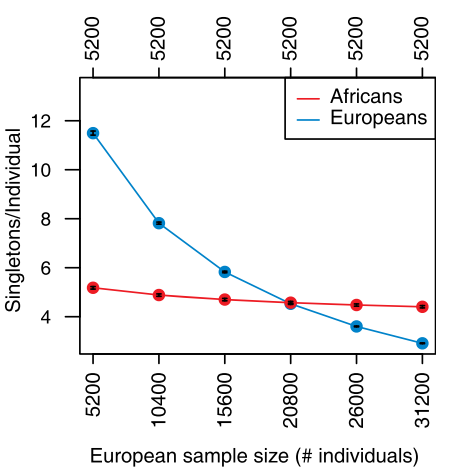

Fig. 1 Simulated numbers of singletons per individual across sample size and ratio in Africans and Europeans. a The number of simulated singletons per individual is shown for Africans (red) and Europeans (blue) at different population sizes. Each panel has a different constant ratio of African to European sample size. Black error bars represent a 95\% confidence interval from 200 replicates. $\mathbf{b}$ The number of simulated singletons per individual is shown for Africans (red) and Europeans (blue). However, African sample size is held constant in each panel, with African to European sample size ratio varying along the $x$-axis. Black error bars represent a 95\% confidence interval from 200 replicates 


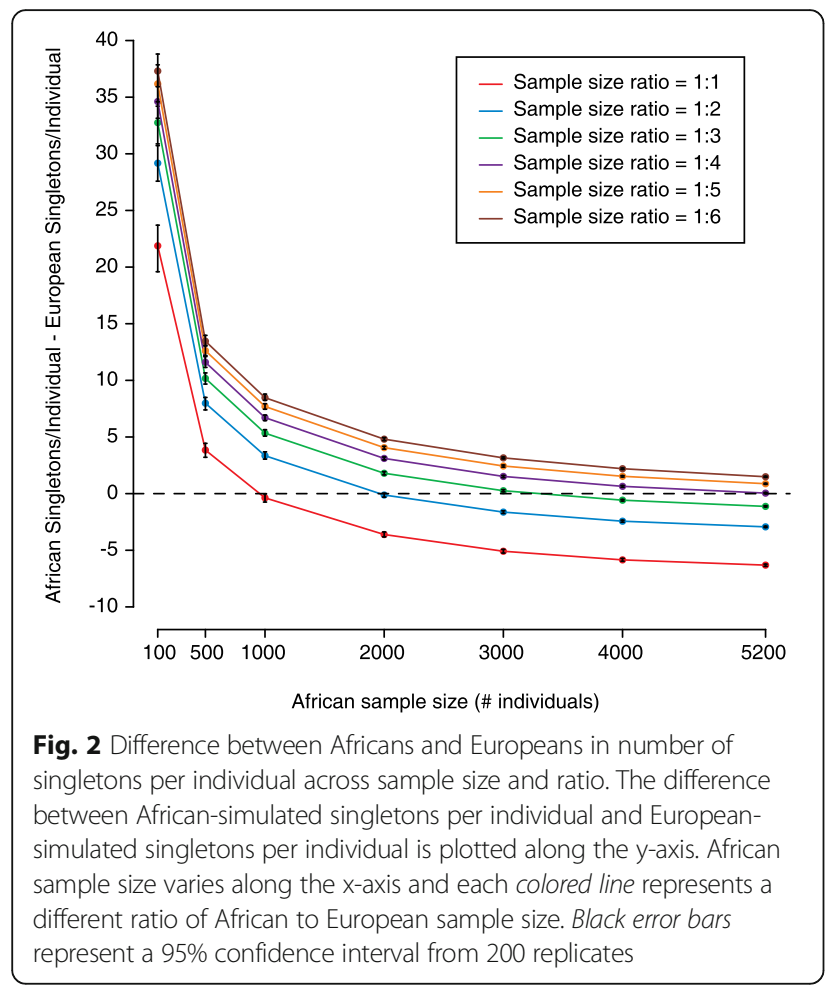

This impact of sample size and ratio can help to explain the difference across ancestry in singletons per individual seen by Petrovski and Goldstein [1]. In their initial analysis, the ratio of African to European sample size was 1:10.05 and the sample sizes themselves were relatively small (505 Africans and 5094 Europeans). When they included the ExAC reference data set in their analysis, the population size ratio increased to $1: 6.74$ and the overall sizes also grew (to 5708 Africans and 38,464 Europeans). Our simulations show that both of these changes will reduce the number of singletons in African individuals by more than that in European individuals, as is seen in the results published by Petrovski and Goldstein [1]. However, had the ExAC data included in the analysis resulted in the appropriate sample size and/ or ratio, the pattern they highlight would have disappeared or even reversed. While we strongly agree with the need to increase the representation of nonEuropeans in sequencing studies and the need to further understand the impact of ancestry-specific genomic patterns [8], our results support the need to consider population-specific allele distributions (i.e., site frequency spectra) when establishing population proportions within a study. By doing this, differences between Europeans and underrepresented populations can potentially be addressed without the inclusion of equal numbers of samples from each population. Overall, we applaud the efforts of Petrovski and Goldstein to highlight the need to make resources equally useful to all. In order to take a significant step forward in reaching this goal, we must account for the impact of demographic history and how it shapes inter-population differences in allele frequency distributions.

\section{Additional file}

Additional file 1: Supplementary note and figures. The supplementary note describes the simulations we performed and the figures are analogous to the main text figures but represent data from simulations done with Latino, South Asian, and East Asian allele frequencies. (PDF 666 kb)

\section{Abbreviations}

ExAC: Exome Aggregation Consortium; OMIM: Online Mendelian Inheritance in Man

\section{Acknowledgements}

The authors thank Wei Song, Amol Shetty, and Daniel Harris for thoughtful discussion and insightful feedback. Funding for this study was provided by the Center for Health Related Informatics and Bioimaging at the University of Maryland.

\section{Authors' contributions}

MDK conceived the project. MDK performed the experiments. MDK and TDO designed the experiments, analyzed the data, and wrote the manuscript. Both authors read and approved the final manuscript.

\section{Competing interests}

The authors declare that they have no competing interest.

\section{Author details}

${ }^{1}$ Institute for Genome Sciences, University of Maryland School of Medicine, Baltimore, MD 21201, USA. ²Department of Medicine, University of Maryland School of Medicine, Baltimore, MD 21201, USA. ${ }^{3}$ Program in Personalized and Genomic Medicine, University of Maryland School of Medicine, Baltimore, MD 21201, USA. ${ }^{4}$ University of Maryland Marlene and Stewart Greenebaum Comprehensive Cancer Center, Baltimore, MD 21201, USA.

Published online: 27 February 2017

\section{References}

1. Petrovski S, Goldstein DB. Unequal representation of genetic variation across ancestry groups creates healthcare inequality in the application of precision medicine. Genome Biol. 2016;17:157.

2. Amberger JS, Bocchini CA, Schiettecatte F, Scott AF, Hamosh A. OMIM.org: Online Mendelian Inheritance in Man (OMIM(R)), an online catalog of human genes and genetic disorders. Nucleic Acids Res. 2015;43:D789-98.

3. Lek M, Karczewski KJ, Minikel EV, Samocha KE, Banks E, Fennell T, et al. Analysis of protein-coding genetic variation in 60,706 humans. Nature. 2016;536:285-91.

4. Tennessen JA, Bigham AW, O'Connor TD, Fu W, Kenny EE, Gravel S, et al. Evolution and functional impact of rare coding variation from deep sequencing of human exomes. Science. 2012;337:64-9.

5. Fu W, O'Connor TD, Jun G, Kang HM, Abecasis G, Leal SM, et al. Analysis of 6,515 exomes reveals the recent origin of most human protein-coding variants. Nature. 2013;493:216-20.

6. 1000 Genomes Project, Auton A, Brooks LD, Durbin RM, Garrison EP, Kang HM, et al. A global reference for human genetic variation. Nature. 2015;526:68-74.

7. Gravel S, Henn BM, Gutenkunst RN, Indap AR, Marth GT, Clark AG, et al. Demographic history and rare allele sharing among human populations. Proc Natl Acad Sci U S A. 2011;108:11983-8.

8. Kessler MD, Yerges-Armstrong L, Taub MA, Shetty AC, Maloney K, Jeng LJ, et al. Challenges and disparities in the application of personalized genomic medicine to populations with African ancestry. Nat Commun. 2016;7:12521. 